\title{
SINTOMATOLOGÍA DEPRESIVA Y PROBLEMAS RELACIONADOS AL CONSUMO DE ALCOHOL DURANTE LA FORMACIÓN ACADÉMICA DE ESTUDIANTES DE MEDICINA
}

\author{
Rubén Valle ${ }^{1, a}$, Elard Sánchez $2, b$, Alberto Perales $2, b$
}

\author{
RESUMEN
}

Con el objetivo de evaluar la frecuencia de sintomatología depresiva (SDe) y problemas relacionados al consumo de alcohol (PRCA) durante la formación académica de estudiantes de Medicina de la Universidad Nacional Mayor de San Marcos, se realizó un estudio transversal en estos estudiantes, del primero a sexto año. Usando la escala de depresión de Zung, para evaluar SDe, y el cuestionario CAGE, para evaluar PRCA, se encontró que el 23,3\% de los encuestados presentó SDe y el 7,3\%, PRCA. Se encontró, así mismo que la frecuencia de SDe y PRCA fue mayor en los estudiantes de los primeros años de estudios. Se recomienda que hay necesidad de actuar en la prevención y detección de estas entidades desde los primeros años de formación académica de estudiantes de Medicina.

Palabras clave: Depresión; Trastorno depresivo; Consumo de bebidas alcohólicas; Estudiantes de medicina; Educación de pregrado de medicina (fuente: DeCS BIREME).

\section{DEPRESSIVE SYMPTOMATOLOGY AND ALCOHOL-RELATED PROBLEMS DURING THE ACADEMIC TRAINING OF MEDICAL STUDENTS}

\begin{abstract}
In order to evaluate the frequency of depressive symptomatology (DS) and alcohol-related problems (ARP) during the academic training of medical students from Universidad Nacional Mayor de San Marcos, a cross-sectional study was conducted among students from first to sixth year of career. The Zung Self-Rating depression scale was used to evaluate DS and the CAGE questionnaire to evaluate ARP. $23.3 \%$ of participants had DS, and $7.3 \%$ had ARP. We found that the frequency of DS and ARP was higher among students in the first years of career. We recommend it is necessary to take action in the prevention and detection of these disorders from the first years of training of medical students.
\end{abstract}

Key words: Depression; Depressive disorder; Alcohol drinking; Alcohol-Related Disorders, Students, medical; Education, medical, undergraduate (source: MeSH NLM).

\section{INTRODUCCIÓN}

La depresión es uno de los trastornos clínicos más frecuentes en la población general; sin embargo, numerosos estudios han probado que este trastorno puede ser aun más frecuente en determinados grupos especialmente vulnerables. Los estudiantes universitarios se encuentran permanentemente sometidos a diversos estresores (académicos, económicos, familiares, laborales, entre otros); se conoce que la prevalencia de sintomatología depresiva (SDe) en estudiantes universitarios de Lima Metropolitana es de $13 \%{ }^{(1)}$; se ha informado además que en estudiantes de ciencias de la salud y estudiantes de Medicina estas prevalencias pueden incrementarse hasta 31,2 y $33,6 \%$, respectivamente (2). Por otro lado, los problemas relacionados al consumo de alcohol (PRCA), entendiendo este como el consumo de alcohol que implica consecuencias negativas a la persona o en su entorno social ${ }^{(3)}$, también han sido descritos en estudiantes de Medicina, encontrando prevalencias en estudiantes de primer y último año de 36 y $18 \%$ respectivamente ${ }^{(4)}$.

\footnotetext{
Facultad de Medicina Humana "San Fernando", Universidad Nacional Mayor de San Marcos. Lima, Perú.

Instituto de Ética en Salud, Facultad de Medicina Humana "San Fernando", Universidad Nacional Mayor de San Marcos. Lima, Perú.

Médico cirujano, ${ }^{\mathrm{b}}$ médico psiquiatra

Recibido: 02-08-12 Aprobado: 23-01-13
}

Citar como: Valle R, Sánchez E, Perales A. Sintomatología depresiva y problemas relacionados al consumo de alcohol durante la formación académica de estudiantes de medicina. Rev Peru Med Exp Salud Publica. 2013;30(1):54-7. 
Con relación al tiempo de aparición de estos trastornos, los estudios muestran resultados variables, e incluso contradictorios, hay quienes afirman que la prevalencia de SDe y PRCA es mayor en los primeros años de estudios en comparación con lo observado al final de su carrera ${ }^{(4,5)}$; mientras que otros señalan que la prevalencia de depresión en estudiantes al inicio de sus carreras es similar a la población general, para luego incrementar y mantenerse elevadas a través del tiempo ${ }^{(6,7)}$. En relación al alcohol, se ha señalado que el consumo es similar durante los años de estudios preclínicos y clínicos ${ }^{(8)}$

Más importante aun, es que a pesar de la alta prevalencia de estos trastornos existe un bajo número de consultas médicas en los servicios de salud mental; un estudio encontró que solo el $22 \%$ de aquellos estudiantes universitarios con cuadros depresivos acudieron a algún servicio de salud mental en busca de ayuda ${ }^{(9)}$. Por este motivo, se ha resaltado la importancia de realizar pruebas de tamizaje durante la formación universitaria y en los servicios de salud como una medida para detectar a individuos que podrían padecer de alguno de estos trastornos ${ }^{(10)}$, para poder guiarlos hacia la búsqueda de una atención oportuna. El presente trabajo busca evaluar la frecuencia de SDe y PRCA en estudiantes de Medicina de la Universidad Nacional Mayor de San Marcos (UNMSM) durante el año 2010.

\section{EL ESTUDIO}

Se realizó un análisis secundario de los datos de un estudio transversal desarrollado en la Facultad de Medicina de la UNMSM (Lima-Perú) que tuvo como objetivo principal estudiar la conducta suicida en estudiantes de Medicina durante el primer semestre del año 2010, con la finalidad de sugerir la implementación de un programa de evaluación y soporte en salud mental para estos estudiantes (11). En dicho trabajo, además, se evaluaron enfermedades de salud mental que pudieran estar asociadas a la conducta objetivo, entre ellas, SDe y PRCA, las cuales fueron consideradas para el presente estudio.

Durante el año académico 2010, en la Facultad de Medicina de la UNMSM, se encontraban matriculados 919 estudiantes, los cuales constituyen nuestra población. Las encuestas se realizaron a manera de censo, procurando realizarlas durante las clases magistrales de los cursos a los que debían asistir la totalidad del alumnado de cada año en estudio.

Para evaluar los SDe se empleó la escala de depresión de Zung, la cual se encuentra validada en el Perú ${ }^{(12)}$, ella consta de 20 preguntas y recoge información de manera cuantitativa (de 20 a 80 puntos), un puntaje mayor a 50 puntos fue considerado como presencia de depresión. Se usó el cuestionario CAGE (del acrónimo en inglés: Cut-down, Annoyed, Guilty, Eye-opener) (13) para evaluar los PRCA, el cual es una escala de cero a cuatro puntos, la presencia de PRCA fue definida como un puntaje mayor o igual a 2 .

Para la recolección de datos se construyó un instrumento, el cual además de recoger la información sociodemográfica de los participantes, incluyó la escala Zung, el cuestionario CAGE y el consentimiento informado. La encuesta fue voluntaria, autoaplicada y tuvo una duración de $30 \mathrm{~min}$, cada participante debió consignar su nombre en la encuesta.

El análisis de los datos incluyó estadística descriptiva. Para el análisis de los datos cualitativos se empleó media con desviación estándar; en tanto que para las variables categóricas se determinó las frecuencias relativas y absolutas. Para ello se empleó el paquete estadístico STATA V.12.0.

El estudio de evaluación original cuenta con la aprobación del Comité de Ética de la UNMSM y por el Vicerrectorado de Investigación de esta casa de estudios (Código: PEM2009C54) (11); esta aprobación contempló que cada participante se identificará con la finalidad de ofrecer orientación terapéutica a quienes así lo requieran, los autores de ambos estudios garantizaron la confidencialidad de esta información. El análisis secundario, al compartir los objetivos con el estudio original, no requirió de una segunda aprobación. Previo a la aplicación del cuestionario se explicó a los participantes los objetivos del estudio, haciendo énfasis que este era voluntario, aquellos que decidieron participar firmaron un consentimiento informado.

\section{HALLAZGOS}

Se logró encuestar al 66,9\% (615/919) de la población estudiantil, el 33,1\% (304/919) estudiantes decidieron no participar del estudio. Entre los encuestados el 58\% (357/615) fueron de sexo masculino, la media de edad de la población fue de $22 \pm 4.5$ años; el $78 \%$ vivía con su familia nuclear; el $74 \%$ procedía de Lima; el 99\% era soltero; el 2,1\% mencionó tener al menos un hijo, y el $10 \%$ informó que estudiaba y trabajaba simultáneamente.

La frecuencia de SDe en la población estudiada fue de $23,3 \%$ (143/615), la frecuencia en los tres primeros años fue $29,6 \%$ y en aquellos de los tres últimos años, $14,6 \%$, pudiéndose apreciar que la frecuencia de SDe disminuía conforme aumentaron los años académicos. El 7,3\% (45/615) presentó PRCA, la frecuencia de PRCA fue mayor en los tres primeros años (8,7\%) en comparación 
Tabla 1. Sintomatología depresiva y problemas relacionados al consumo de alcohol en estudiantes de Medicina de la Universidad Nacional Mayor de San Marcos, 2010.

\begin{tabular}{|c|c|c|c|}
\hline & & $\begin{array}{c}\text { Sintomatología } \\
\text { depresiva }\end{array}$ & $\begin{array}{c}\text { Problemas } \\
\text { relacionados } \\
\text { al consumo de } \\
\text { alcohol }\end{array}$ \\
\hline & $\mathbf{N} .^{\circ}$ & N. ${ }^{\circ}(\%)$ & $\mathrm{N} .{ }^{\circ}(\%)$ \\
\hline \multicolumn{4}{|l|}{ Sexo } \\
\hline Femenino & 258 & $75(29,1)$ & $10(3,9)$ \\
\hline Masculino & 357 & $68(19,1)$ & $35(9,8)$ \\
\hline \multicolumn{4}{|l|}{ Edad $^{\dagger}$} \\
\hline 16-21 años & 305 & $83(27,2)$ & $25(8,2)$ \\
\hline >21 años & 301 & $59(19,6)$ & $17(5,7)$ \\
\hline \multicolumn{4}{|c|}{ Procedente de provincia } \\
\hline Sí & 72 & $19(26,4)$ & $7(9,7)$ \\
\hline No & 543 & $124(22,8)$ & $38(7)$ \\
\hline \multicolumn{4}{|l|}{ Hijos } \\
\hline Sí & 12 & $3(25)$ & $1(8,3)$ \\
\hline No & 544 & $125(22,9)$ & $40(7,4)$ \\
\hline \multicolumn{4}{|c|}{ Año de estudio } \\
\hline Primero & 139 & $53(38,1)$ & $12(8,6)$ \\
\hline Segundo & 135 & $33(24,4)$ & $11(8,2)$ \\
\hline Tercero & 81 & $19(23,5)$ & $8(9,9)$ \\
\hline Cuarto & 89 & $12(13,5)$ & $3(3,4)$ \\
\hline Quinto & 114 & $16(14)$ & $7(6,1)$ \\
\hline Sexto & 57 & $10(17,5)$ & $4(7)$ \\
\hline \multicolumn{4}{|l|}{ Trabaja } \\
\hline Sí & 63 & $14(22,2)$ & $7(11,1)$ \\
\hline No & 552 & $129(23,4)$ & $38(6,9)$ \\
\hline \multicolumn{4}{|l|}{ Vive solo } \\
\hline Sí & 32 & $9(28,1)$ & $2(6,3)$ \\
\hline No & 581 & $133(22,9)$ & $43(7,4)$ \\
\hline \multicolumn{4}{|c|}{ Familia nuclear } \\
\hline Sí & 479 & $109(22,8)$ & $34(7,1)$ \\
\hline No & 134 & $33(24,6)$ & $11(8,2)$ \\
\hline
\end{tabular}

† Se formaron dos grupos en función a la mediana (21 años).

Nota: la suma de las categorías puede no ser la misma en todas las variables debido a que no se eliminaron los registros incompletos.

a los tres últimos años (5,3\%) e igualmente se observó que su frecuencia disminuía conforme aumentaron los años académicos (Tabla 1 ).

\section{DISCUSIÓN}

Nuestro estudio pone en evidencia la alta frecuencia de SDe en estudiantes de Medicina en comparación con la población general; pues la prevalencia de SDe para Lima Metropolitana, en este mismo grupo de edad es de $10,3 \%{ }^{(14)}$. Ello concuerda con lo informado en universidades privadas de Lima que reportaron prevalencias de SDe de 34,1 y 33,6\% (2,15); y con lo informado en Brasil, en donde se encontró una prevalencia de $38,2 \%{ }^{(5)}$; sin embargo, existen otros estudios que han encontrado prevalencias menores a la población general. En Corea del Sur, por ejemplo, se ha descrito una prevalencia de SDe de $9,4 \%$ en estudiantes de medicina ${ }^{(16)}$

Nuestros resultados son consistentes con aquellos que mencionan que la prevalencia de SDe es mayor en los primeros años de estudio ${ }^{(15,16)}$. Este patrón de presentación podría ser el resultado de la adaptación a los estresores de la vida universitaria que afronta el estudiante, lo cual, sumado a la presencia de problemas individuales y a la ausencia de un sistema de soporte personal, podría explicar el desarrollo de SDe en aquellos con mayor vulnerabilidad.

En relación a la mayor prevalencia de SDe en estudiantes de los primeros años, se ha sugerido que existiria un mayor riesgo entre los postulantes a las facultades de Medicina ${ }^{(17)}$; sin embargo, otro estudio sugiere que los estudiantes de Medicina de los primeros años no son diferentes de aquellos de otras carreras profesionales ${ }^{(7)}$. Consideramos que son los estresores propios de la actividad académica y los problemas individuales los responsables de la mayor presencia de SDe en estudiantes de Medicina de los primeros años de estudios.

Respecto al consumo de alcohol, nuestros resultados son similares a otra investigación que encontró una prevalencia de PRCA de 8,5 y $4,8 \%$ en estudiantes de primer y sexto año, respectivamente ${ }^{(17)}$. En nuestro estudio, la frecuencia de PRCA disminuyó conforme aumentan los años de estudios. Consideramos que el consumo de alcohol podría estar relacionado a las nuevas oportunidades sociales, el poder de decisión y la presión del medio social hacia el consumo de alcohol ${ }^{(8)}$. Por otro lado, la disminución de la frecuencia de los PRCA en los años posteriores, puede ser consecuencia de una mayor adaptabilidad de los estudiantes al estrés de la carrera universitaria y sobre todo al mayor conocimiento que pueden tener acerca de las consecuencias del consumo de alcohol.

Creemos que los SDe y PRCA constituyen trastornos de la salud mental que tienen una alta prevalencia entre los estudiantes de Medicina; por este motivo, creemos que la aplicación de pruebas de tamizaje, adecuadamente validadas, resultaría de utilidad para la identificación temprana de estos trastornos en los estudiantes. Considerando esta situación, diferentes estudios señalan la importancia de implementar programas de soporte de salud mental ${ }^{(4,16)}$, con un enfoque preventivo y terapéutico y que abarquen los factores personales y contextuales que pueden desencadenar estos trastornos. Reconocemos que estas escalas no sustituyen a la evaluación psiquiátrica, pero al tener una alta correlación con el diagnóstico clínico sirven 
para identificar a los individuos con alto riesgo, y así poder ayudarlos a tiempo. En la Facultad de Medicina de la UNMSM se viene implementando esta medida de intervención a través del "Preventorio para el Desarrollo Humano" (Proyecto Aprobado por RD 01498-D-FM2011, Reglamento Aprobado por RD 0904-D-FM-2012).

Finalmente, algunas limitaciones del trabajo deben ser reconocidas. Un alto porcentaje de estudiantes (33\%) no pudo ser encuestado, lo que constituye un sesgo de selección, cabe la posibilidad de que algunos estudiantes hayan decidido no participar dado que padecían algunas de las entidades en estudio. No se puede descartar la posibilidad que exista un sesgo de medición, dado que el cuestionario no fue anónimo. Por consiguiente, es probable que las frecuencias encontradas en nuestro estudio sean menores a las reales. Por último, debido a que se empleó un muestreo no probabilístico, no se pudo realizar inferencia estadística de los datos.

En conclusión, existe una alta frecuencia de SDe y PRCA en estudiantes de Medicina de la UNMSM en los primeros años de la carrera. Creemos que nuestros resultados constituyen un primer soporte técnico y científico para la implementación de programas de salud mental en las escuelas de Medicina, los cuales podrian detectar tempranamente problemas de salud mental en los estudiantes y evitar posibles complicaciones futuras.

Contribuciones de autoría: AP participó de la concepción, diseño del estudio, recolección de los datos, revisión crítica del artículo y obtención del financiamiento. RV participó del análisis e interpretación de los datos, redacción del artículo, revisión crítica del estudio. ES participó de la recolección y obtención de los datos así como de la revisión crítica del artículo. Todos los revisores aprobaron la versión final del artículo.

Fuentes de financiamiento: artículo derivado del proyecto financiado por el Vicerrectorado de Investigación de la UNMSM. Código: PEM2009C54, aprobado con RR 00071-R10 del 18 de enero de 2010.

Conflictos de interés: los autores niegan conflictos de intereses.

\section{REFERENCIAS BIBLIOGRÁFICAS}

1. Riveros M, Hernádez H, Rivera J. Niveles de depresión y ansiedad en estudiantes universitarios de Lima metropolitana. Revista de Investigación en Psicología. 2007;10(1):91-102.

2. Pereyra-Elías R, Ocampo-Mascaró J, Silva-Salazar V, Vélez-Segovia E, Costa-Bullón AD da, Toro-Polo LM, et al. Prevalencia y factores asociados con síntomas depresivos en estudiantes de ciencias de la salud de una Universidad privada de Lima, Peru 2010. Rev Peru Med Exp Salud Publica. 2010;27(4):520-6.

3. Comisión Nacional para el Desarrollo y Vida sin Drogas (DEVIDA). III Estudio Nacional: Prevención y consumo de drogas en estudiantes de educación secundaria 2009. Lima: DEVIDA; 2011.

4. Granville-Chapman JE, Yu K, White PD. A follow-up survey of alcohol consumption and knowledge in medical students. Alcohol Alcohol. 2001;36(6):540-3.

5. Baldassin S, Alves TC, de Andrade AG, Nogueira Martins LA. The characteristics of depressive symptoms in medical students during medical education and training: a cross-sectional study. BMC Med Educ. 2008;8:60.

6. Schwenk TL, Davis L, Wimsatt LA. Depression, stigma, and suicidal ideation in medical students. JAMA. 2010;304(11):1181-90.
7. Rosal MC, Ockene IS, Ockene JK, Barrett SV, Ma Y, Hebert JR. A longitudinal study of students' depression at one medical school. Acad Med. 1997;72(6):542-6.

8. Deressa W, Azazh A. Substance use and its predictors among undergraduate medical students of Addis Ababa University in Ethiopia. BMC Public Health. 2011;11:660.

9. Givens JL, Tjia J. Depressed medical students' use of mental health services and barriers to use. Acad Med. 2002;77(9):918-21.

10. Dawson DA, Goldstein RB, Grant BF. Factors associated with first utilization of different types of care for alcohol problems. J Stud Alcohol Drugs. 2012;73(4):647-56.

11. Perales A, Alarcón JO, Sánchez E, Arcaya MJ, Cortez E, Parhuana A, et al. Conducta suicida en estudiantes de medicina de una universidad nacional peruana. Informe Final. Código: PEM2009C54. Lima: Universidad Nacional Mayor de San Marcos; 2011.

12. Warthon DD, Novara J, Sotillo C. Estandarización y correlación de las escalas de Beck, Hamilton y Zung para la depresión en la población de Lima Metropolitana. Lima: INSM-HDHN; 1985.

13. Bobes-García J, García-Portilla MP, Bascarán-Fernández MT, Sáiz-Martínez PA,
Bousoño-García M. Banco de instrumentos básicos para la práctica de la psiquiatría clínica. 3rd ed. Barcelona: Ars Medica; 2004.

14. Instituto Nacional de Salud Mental Honorio Delgado-Hideyo Noguchi. Estudio Epidemiológico Metropolitano de Salud Mental 2002. Anales de Salud Mental. 2002;18(1-2):13-153.

15. Osada J, Rojas M, Rosales C, VegaDienstmaier JM. Sintomatología ansiosa y depresiva en estudiantes de medicina. Rev Neuropsiquiatr. 2010;73(1):15-9.

16. Roh MS, Jeon HJ, Kim H, Cho HJ, Han SK, Hahm BJ. Factors influencing treatment for depression among medical students: a nationwide sample in South Korea. Med Educ. 2009;43(2):133-9.

17. Akvardar Y, Demiral Y, Ergor G, Ergor A. Substance use among medical students and physicians in a medical school in Turkey. Soc Psychiatry Psychiatr Epidemiol. 2004;39(6):502-6.

Correspondencia: Rubén Valle Rivadeneyra Dirección: Jr. Filadelfia 2365. Lima 21, Perú Teléfono: (511) 985835737

Correo electrónico: ruben_vr12@hotmail.com 